\title{
Anti-Inflammatory Effects of 81 Chinese Herb Extracts and Their Correlation with the Characteristics of Traditional Chinese Medicine
}

\author{
Chang-Liang Chen and Dan-Dan Zhang \\ Shanghai University of Traditional Chinese Medicine, Pudong, Shanghai 201203, China
}

Correspondence should be addressed to Dan-Dan Zhang; izhangdd@126.com

Received 26 November 2013; Accepted 13 January 2014; Published 20 February 2014

Academic Editor: Yemeng Chen

Copyright (c) 2014 C.-L. Chen and D.-D. Zhang. This is an open access article distributed under the Creative Commons Attribution License, which permits unrestricted use, distribution, and reproduction in any medium, provided the original work is properly cited.

\begin{abstract}
Inducible nitrogen oxide synthase (iNOS) is the primary contributor of the overproduction of nitric oxide and its inhibitors have been actively sought as effective anti-inflammatory agents. In this study, we prepared $70 \%$ ethanol extracts from 81 Chinese herbs. These extracts were subsequently evaluated for their effect on nitrogen oxide (NO) production and cell growth in LPS/IFN $\gamma$ costimulated and unstimulated murine macrophage RAW264.7 cells by Griess reaction and MTT assay. Extracts of Daphne genkwa Sieb.et Zucc, Caesalpinia sappan L., Iles pubescens Hook.et Arn, Forsythia suspensa (Thunb.) Vahl, Zingiber officinale Rosc, Inula japonica Thunb., and Ligusticum chuanxiong Hort markedly inhibited NO production (inhibition $>90 \%$ at $100 \mu \mathrm{g} / \mathrm{mL}$ ). Among active extracts (inhibition $>50 \%$ at $100 \mu \mathrm{g} / \mathrm{mL}$ ), Rubia cordifolia L., Glycyrrhiza glabra L., Iles pubescens Hook.et Arn, Nigella glandulifera Freyn et Sint, Pueraria lobata (Willd.) Ohwi, and Scutellaria barbata D. Don displayed no cytotoxicity to unstimulated RAW246.7 cells while increasing the growth of LPS/IFN $\gamma$-costimulated cells. By analyzing the correlation between their activities and their Traditional Chinese Medicine (TCM) characteristics, herbs with pungent flavor displayed potent anti-inflammatory capability. Our study provides a series of potential anti-inflammatory herbs and suggests that herbs with pungent flavor are candidates of effective anti-inflammatory agents.
\end{abstract}

\section{Introduction}

Inflammation is a self-protection mechanism aiming at removing harmful stimuli, including damaged cells, irritants, or pathogens, and beginning the wound repair process. However, inflammation sometimes induces further inflammation, leading to self-perpetuating chronic inflammation that can cause severe cellular injury and tissue damage [1]. Chronic inflammation has been linked to a wide variety of diseases such as atherosclerosis [2], Alzheimer's disease [3], diabetes [4], and carcinogenesis [5-7].

Nitric oxide (NO), which is mainly generated by inducible nitric oxide synthase (iNOS) under the inflammatory conditions [8-10], plays a key role in each step of the pathological processes during inflammation [11-14]. Selective inhibitors of iNOS have been shown to be both anti-inflammatory and tissue-protective in various inflammatory animal models [1517] and are thus regarded as promising agents for treating inflammatory diseases. High expression of iNOS can often be detected in human tumors, supporting the notion that chronic inflammation is actively involved in tumor progression [18-21]. In fact, nonsteroidal anti-inflammatory drugs (NSAIDs), including aspirin [22] and tolfenamic acid [23], are currently used for both cancer prevention and treatment [24].

A variety of natural products have been reported to possess anti-inflammatory and anticancer effects in experimental animal models. For example, curcumin has been shown to inhibit cyclooxygenase 2 (COX2) expression and is actually in clinical use as a chemoprevention agent [2527]. Because of the promises in curcumin, extensive efforts have also been exerted to identify compounds capable of targeting inflammatory mediators [28-30]. A recent study by Liao et al. investigated the potential association between antioxidation capability and the characteristics of Traditional Chinese Medicine (TCM) in 45 commonly used Chinese 
herbs, in which antioxidation capability of Chinese herbs was found to be correlated with their flavor characteristics [31]. Their findings are very encouraging because it indicates that effective anti-inflammatory agents may potentially be identified from Chinese herbs based on their TCM characteristics.

In our effort to identify effective anti-inflammatory agents, we prepared $70 \%$ ethanol extracts from 81 Chinese herbs and subsequently tested their abilities to suppress NO production in murine macrophage RAW264.7 cells costimulated with LPS and IFN $\gamma$. Moreover, we also analyzed the correlation between anti-inflammatory capacity and TCM characteristics among these herbs. We conclude that herbs with pungent flavor are the strongest in their antiinflammatory capability.

\section{Materials and Methods}

2.1. Chemicals. IFN $\gamma$ was purchased from EMD Millpore Chemicals (Billerica, MA, USA). Bovine serum albumin (BSA), lipopolysaccharide (LPS, E. coli 0111: B4), N(1-naphthyl)-ethylenediamine dihydrochloride (L-NIL), 3(4, 5-dimethylthiazol-2-yl)-2, 5-diphenyltetrazolium (MTT), naphthylethylenediamine, sulfanilamide, and sodium bicarbonate were all obtained from Sigma-Aldrich Co (St. Louis, MO, USA). RPMI 1640 and trypsin-EDTA were purchased from Life Technologies (Grand Island, NY, USA). Fetal bovine serum (FBS) was purchased from Hyclone Thermo Fisher Scientific (Waltham, MA, USA).

2.2. Preparation of $70 \%$ Ethanol Extracts of Chinese Herbs. All herbs were obtained from YANG He Tang and Kangqiao Co (Shanghai, China). All 81 herbs chosen for our study have been reported or suggested to have potential antiinflammatory activities by either TCM literatures or current pharmacological reports. Botanical identification of these herbs was performed by Shanghai Institute for Food and Drug Control (SIFDC). To prepare ethanol extracts, $100 \mathrm{~g}$ of each dried herbs was sliced and extracted with $1 \mathrm{~L}$ of $70 \%$ ethanol at $80^{\circ} \mathrm{C}$ for three times. Obtained ethanol extracts were evaporated under reduced pressure at temperature $60^{\circ} \mathrm{C}$ and stored at $-80^{\circ} \mathrm{C}$. Extracts were dissolved with DMSO before use.

2.3. Measurement of Nitrite Production. RAW264.7 cells were plated in 96 -well plates $\left(5 \times 10^{3}\right.$ cells per well) for overnight and then replenished with FBS-free medium for $10 \mathrm{~h}$ followed by adding $100 \mu \mathrm{g} / \mathrm{mL}$ herb extracts into each well. Cells were costimulated with $100 \mathrm{ng} / \mathrm{mL}$ LPS and $10 \mathrm{U} / \mathrm{mL}$ IFN $\gamma$ for $24 \mathrm{~h}$, and media were then collected and analyzed for the amount of nitrite, a stable oxidative metabolite and faithful NO indicator, by the Griess reaction as previously described [32]. To do it, $100 \mu \mathrm{L}$ of Griess reagent $(0.1 \%$ naphthyl-ethylenediamine and $1 \%$ sulfanilamide in $5 \%$ phosphoric acid) was mixed with $100 \mu \mathrm{L}$ of collected medium in a 96-well plate. Mixture was incubated for $10 \mathrm{~min}$ at room temperature and then read at $540 \mathrm{~nm}$. The amount of nitrite was calculated based on a standard curve generated with sodium nitrite. Percent inhibition in NO production was calculated with the formula $\{[$ (nitrite with herb extract) - (nitrite without herb extract) $] /$ (nitrite without herb extract) $\} \times 100$.

2.4. Analysis of Cell Viability. Cell viability was determined by MTT assay as previously described [33]. Briefly, RAW264.7 cells were incubated with MTT $(5 \mathrm{mg} / \mathrm{mL}$ in phosphatebuffered saline, $\mathrm{pH}=7.4$ ) for $4 \mathrm{~h}$. Formed MTT formazan was solubilized with $50 \mu \mathrm{L}$ of $0.01 \mathrm{M} \mathrm{HCl}$ buffer containing $10 \%$ SDS and 5\% isobutanol. Cell growth was determined by reading plates at $570 \mathrm{~nm}$ in a microplate reader. The cell viability of control group is considered as $100 \%$.

2.5. Statistical Analysis. The direction and magnitude of correlation between variables was done using analysis of $t$-test. $P$ values less than 0.05 were considered statistically significant $\left({ }^{*} P<0.05\right)$.

\section{Results}

3.1. Effect of Herb Extracts on NO Production and Cell Growth. With the aid of Griess assay, we analyzed ethanol extracts of 81 herbs for their anti-inflammatory activity. A wide range of inhibition in NO production was observed with these extracts (Table 1). Extracts of 7 herbs [Daphne genkwa Sieb.et Zucc, Caesalpinia sappan L., Iles pubescens Hook.et Arn, Forsythia suspensa (Thunb.) Vahl, Zingiber officinale Rosc, Inula japonica Thunb., and Ligusticum chuanxiong Hort] blocked over $90 \%$ NO production in LPS/IFN $\gamma$-stimulated RAW264.7 cells (Table 1). Among the extracts that elicited over $50 \%$ inhibition in NO production, Rubia cordifolia L., Glycyrrhiza glabra L., Iles pubescens Hook.et Arn, Nigella glandulifera Freyn et Sint, Pueraria lobata (Willd.) Ohwi, and Scutellaria barbata D. Don showed no cytotoxicity to unstimulated RAW264.7 cells while significantly increased the viability of LPS/IFN $\gamma$-stimulated cells (Table 1). However, Daphne genkwa Sieb.et Zucc, which has the strongest inhibitory effect on NO production, was moderately toxic to RAW264.7 cells (Table 1).

3.2. Correlation between Anti-Inflammatory Potency and TCM Characteristics of Herbs. Analyzing the TCM characteristics of 10 herbs that display the strongest inhibitory effect on NO production in LPS/IFN $\gamma$-stimulated RAW264.7 cells, we found that most of them are in the categories of bitter or pungent flavor, warm nature, and lung or liver meridian distributions (Table 2). To correlate the TCM characteristics to anti-inflammatory effect in these herbs, we categorized TCM characteristics of these herbs that were able to abolish $50 \%$ of NO production in LPS/IFN $\gamma$-stimulated RAW264.7 cells. Table 3 showed that herbs with greater antiinflammatory effect were distributed in a significantly higher percentage in those characterized as bitter/pungent flavors, warm nature, and liver/lung meridian distributions. These results suggest that anti-inflammatory herbs may possess 
TABLE 1: Effect of herb extracts on NO production and cell viability in simulated and resting RAW264.7 cells.

\begin{tabular}{|c|c|c|c|c|c|c|}
\hline \multirow[b]{2}{*}{ Plant name and authority } & \multirow[b]{2}{*}{ Part used ${ }^{\mathrm{a}}$} & \multicolumn{2}{|c|}{ Stimulation cells } & \multicolumn{2}{|c|}{ Resting cells } & \multirow[b]{2}{*}{ Yield $^{\mathrm{f}}$} \\
\hline & & $\begin{array}{c}\text { Percent } \\
\text { inhibition of } \\
\mathrm{NO}^{\mathrm{b}}\end{array}$ & $\begin{array}{c}\text { Cell } \\
\text { proliferation } \\
(\%)^{c}\end{array}$ & $\begin{array}{l}\text { NO } \\
\text { production } \\
(\mu \mathrm{M})^{\mathrm{d}}\end{array}$ & $\begin{array}{c}\text { Cytotoxicity } \\
(\%)^{\mathrm{e}}\end{array}$ & \\
\hline Acanthopanax senticosus (Rupr.et Maxim.) Harms & SR & $74.66 \pm 0.01$ & $97.64 \pm 0.09$ & $4.28 \pm 0.01$ & $90.76 \pm 0.04$ & 5.55 \\
\hline Acanthopanax gracilistylus W. W. Smith & $\mathrm{BK}$ & $12.11 \pm 0.01$ & $66.29 \pm 0.01$ & $1.00 \pm 0.01$ & $99.16 \pm 0.01$ & 23.75 \\
\hline Achyranthes bidentata $\mathrm{Bl}$. & RT & $-11.62 \pm 0.02$ & $68.78 \pm 0001$ & $4.13 \pm 0.03$ & $73.25 \pm 0.03$ & 31.25 \\
\hline Acorus tatarinowii Schott. & SR & $11.82 \pm 0.01$ & $47.40 \pm 0.09$ & $0.92 \pm 0.01$ & $98.57 \pm 0.01$ & 17.75 \\
\hline Actinidia arguta (Sieb.et Zucc.) Planch.ex Miq. & RT & $54.67 \pm 0.02$ & $40.79 \pm 0.03$ & $1.18 \pm 0.01$ & $103.69 \pm 0.01$ & 7.42 \\
\hline Actinidia valvata Dunn & RT & $32.11 \pm 0.01$ & $51.09 \pm 0.01$ & $2.94 \pm 0.01$ & $22.07 \pm 0.01$ & 7.36 \\
\hline Alisma orientalis (Sam.) Juzep. & ST & $46.63 \pm 0.04$ & $53.01 \pm 0.02$ & $3.86 \pm 0.02$ & $101.11 \pm 0.04$ & 5.69 \\
\hline Allium macrostemon Bge. & ST & $25.43 \pm 0.03$ & $73.53 \pm 0.04$ & $2.11 \pm 0.01$ & $93.74 \pm 0.02$ & 38.87 \\
\hline Aloe barbadensis Miller & LF & $15.31 \pm 0.10$ & $35.02 \pm 0.02$ & $3.86 \pm 0.02$ & $94.60 \pm 0.04$ & 10.71 \\
\hline Amomum villosum Lour. & FR & $35.56 \pm 0.02$ & $61.15 \pm 0.04$ & $4.57 \pm 0.01$ & $100.19 \pm 0.03$ & 5.18 \\
\hline Artemisia annua L. & $\mathrm{HR}$ & $12.03 \pm 0.03$ & $29.48 \pm 0.03$ & $1.34 \pm 0.01$ & $105.08 \pm 0.01$ & 13.62 \\
\hline Artemisia anomala S. Moore & HR & $59.56 \pm 0.05$ & $74.25 \pm 0.01$ & $1.61 \pm 0.01$ & $102.92 \pm 0.02$ & 11.04 \\
\hline Artemisia capillaris Thunb. & $\mathrm{HR}$ & $41.2 \pm 0.03$ & $64.62 \pm 0.03$ & $4.02 \pm 0.01$ & $104.40 \pm 0.04$ & 17.36 \\
\hline Astragalus membranaceus (Fisch.) Bge. & RT & $13.96 \pm 0.01$ & $53.76 \pm 0.03$ & $4.30 \pm 0.01$ & $93.30 \pm 0.05$ & 47.06 \\
\hline Bambusa tuldoides Munro. & ST & $27.32 \pm 0.01$ & $75.23 \pm 0.01$ & $3.19 \pm 0.01$ & $110.67 \pm 0.03$ & 1.14 \\
\hline Bletilla striata (Thunb.) Reichb. f. & ST & $77.52 \pm 0.01$ & $95.17 \pm 0.08$ & $4.29 \pm 0.01$ & $14.37 \pm 0.02$ & 18.63 \\
\hline Caesalpinia sappan $\mathrm{L}$. & HW & $94.27 \pm 0.01$ & $103.70 \pm 0.01$ & $3.75 \pm 0.01$ & $30.92 \pm 0.01$ & 10.66 \\
\hline Carpesium abrotanoides Linn. & $\mathrm{HR}$ & $74.85 \pm 0.03$ & $53.24 \pm 0.02$ & $2.85 \pm 0.01$ & $102.95 \pm 0.02$ & 11.52 \\
\hline Carthamus tinctorius L. & $\mathrm{FL}$ & $38.89 \pm 0.02$ & $89.78 \pm 0.02$ & $4.22 \pm 0.01$ & $104.26 \pm 0.04$ & 45.76 \\
\hline Celastrus orbiculatus Thunb. & $\mathrm{RT}$ & $-6.06 \pm 0.01$ & $55.62 \pm 0.01$ & $1.30 \pm 0.01$ & $101.88 \pm 0.03$ & 2.78 \\
\hline Cinnamomum cassia Presl. & TW & $38.43 \pm 0.02$ & $86.03 \pm 0.03$ & $4.38 \pm 0.01$ & $107.18 \pm 0.06$ & 9.32 \\
\hline Cinnamomum cassia Presl. & $\mathrm{BK}$ & $68.31 \pm 0.02$ & $97.01 \pm 0.04$ & $4.54 \pm 0.01$ & $60.18 \pm 0.04$ & 11.16 \\
\hline Curcuma longa L. & ST & $89.32 \pm 0.02$ & $108.09 \pm 0.05$ & $4.61 \pm 0.01$ & $51.43 \pm 0.02$ & 9.36 \\
\hline Codonopsis pilosula (Franch.) Nannf. & RT & $-13.92 \pm 0.01$ & $60.45 \pm 0.01$ & $1.42 \pm 0.01$ & $101.83 \pm 0.02$ & 36.04 \\
\hline Corydalis yanhusuo W. T. Wang & ST & $7.36 \pm 0.01$ & $25.78 \pm 0.01$ & $0.74 \pm 0.01$ & $95.26 \pm 0.01$ & 11.14 \\
\hline Chrysanthemum indicum $\mathrm{L}$. & FL & $-2.87 \pm 0.01$ & $97.74 \pm 0.01$ & $1.65 \pm 0.01$ & $101.07 \pm 0.02$ & 26.1 \\
\hline Curculigo orchioides Gaertn. & ST & $8.81 \pm 0.01$ & $29.58 \pm 0.02$ & $1.25 \pm 0.01$ & $106.48 \pm 0.02$ & 8.01 \\
\hline Curcuma wenyujin Y. H. Chen et C. Ling & RT & $-8.31 \pm 0.01$ & $45.33 \pm 0.01$ & $0.59 \pm 0.01$ & $99.01 \pm 0.02$ & 9.423 \\
\hline Curcuma phaeocaulis Val. & ST & $18.87 \pm 0.03$ & $43.53 \pm 0.02$ & $3.10 \pm 0.01$ & $98.00 \pm 0.05$ & 46.14 \\
\hline Dalbergia odorifera T. Chen & HW & $77.38 \pm 0.04$ & $88.27 \pm 0.07$ & $1.93 \pm 0.01$ & $86.12 \pm 0.02$ & 17.6 \\
\hline Daphne genkwa Sieb.et Zucc. & FL & $99.17 \pm 0.01$ & $40.83 \pm 0.03$ & $4.25 \pm 0.01$ & $70.25 \pm 0.04$ & 20.55 \\
\hline Daphne tangutica Maxim. & $\mathrm{BK}$ & $76.12 \pm 0.01$ & $91.32 \pm 0.16$ & $4.56 \pm 0.01$ & $85.67 \pm 0.11$ & 4.75 \\
\hline Drynaria fortunei (Kunze) J. Sm. & ST & $6.46 \pm 0.04$ & $54.58 \pm 0.01$ & $1.15 \pm 0.01$ & $99.59 \pm 0.02$ & 11.458 \\
\hline Epimedium brevicornum Maxim. & LF & $-43.84 \pm 0.02$ & $135.36 \pm 0.02$ & $1.06 \pm 0.01$ & $101.69 \pm 0.02$ & 13.78 \\
\hline Euodia rutaecarpa (Juss.) Benth. & FR & $56.35 \pm 0.02$ & $23.68 \pm 0.01$ & $1.80 \pm 0.01$ & $41.84 \pm 0.03$ & 33.89 \\
\hline Forsythia suspensa (Thunb.) Vahl & FR & $91.93 \pm 0.01$ & $34.44 \pm 0.02$ & $4.24 \pm 0.01$ & $27.32 \pm 0.01$ & 26.12 \\
\hline Gardenia jasminoides Ellis & FR & $15.89 \pm 0.01$ & $50.48 \pm 0.03$ & $4.27 \pm 0.01$ & $129.77 \pm 0.09$ & 29.8 \\
\hline Glycyrrhiza glabra L. & SR & $66.62 \pm 0.01$ & $107.8 \pm 0.07$ & $0.76 \pm 0.01$ & $109.65 \pm 0.03$ & 18.57 \\
\hline Iles pubescens Hook.et Arn. & RT & $65.3 \pm 0.02$ & $106.52 \pm 0.04$ & $4.15 \pm 0.01$ & $117.70 \pm 0.10$ & 7.09 \\
\hline Ilex latifolia Thunb. & $\mathrm{LF}$ & $32.33 \pm 0.09$ & $79.67 \pm 0.01$ & $3.42 \pm 0.01$ & $54.11 \pm 0.04$ & 19.14 \\
\hline Inula japonica Thunb. & $\mathrm{FL}$ & $91.19 \pm 0.01$ & $84.48 \pm 0.03$ & $0.86 \pm 0.01$ & $100.42 \pm 0.01$ & 17.7 \\
\hline Inula linariifolia Turez. & HR & $76.43 \pm 0.01$ & $129.93 \pm 0.19$ & $4.04 \pm 0.01$ & $84.41 \pm 0.03$ & 10.91 \\
\hline Isatis indigotica Fort. & LF & $47.61 \pm 0.02$ & $86.83 \pm 0.05$ & $1.56 \pm 0.01$ & $106.24 \pm 0.02$ & 24.43 \\
\hline Isatis indigotica Fort. & $\mathrm{RT}$ & $26.48 \pm 0.02$ & $53.51 \pm 0.08$ & $1.00 \pm 0.01$ & $103.22 \pm 0.01$ & 26.78 \\
\hline
\end{tabular}


TABle 1: Continued.

\begin{tabular}{|c|c|c|c|c|c|c|}
\hline \multirow[b]{2}{*}{ Plant name and authority } & \multirow[b]{2}{*}{ Part used ${ }^{\mathrm{a}}$} & \multicolumn{2}{|c|}{ Stimulation cells } & \multicolumn{2}{|c|}{ Resting cells } & \multirow[b]{2}{*}{ Yield $^{\mathrm{f}}$} \\
\hline & & $\begin{array}{c}\text { Percent } \\
\text { inhibition of } \\
\mathrm{NO}^{\mathrm{b}}\end{array}$ & $\begin{array}{c}\text { Cell } \\
\text { proliferation } \\
(\%)^{\mathrm{c}}\end{array}$ & $\begin{array}{l}\text { NO } \\
\text { production } \\
(\mu \mathrm{M})^{\mathrm{d}}\end{array}$ & $\begin{array}{l}\text { Cytotoxicity } \\
(\%)^{\mathrm{e}}\end{array}$ & \\
\hline Ligusticum chuanxiong Hort. & SR & $91.13 \pm 0.01$ & $79.46 \pm 0.05$ & $3.88 \pm 0.01$ & $81.82 \pm 0.04$ & 28.1 \\
\hline Lonicera japonica Thunb. & FL & $47.87 \pm 0.02$ & $86.17 \pm 0.04$ & $1.38 \pm 0.01$ & $107.12 \pm 0.01$ & 39.55 \\
\hline Magnolia biondii Pamp. & FL & $-15.35 \pm 0.01$ & $82.89 \pm 0.01$ & $3.27 \pm 0.01$ & $102.22 \pm 0.03$ & 15.39 \\
\hline Morus alba L. & TW & $50.78 \pm 0.01$ & $72.21 \pm 0.06$ & $4.69 \pm 0.01$ & $93.67 \pm 0.03$ & 7.88 \\
\hline Nelumbo nucifera Gaertn. & FR & $21.96 \pm 0.02$ & $95.84 \pm 0.11$ & $4.53 \pm 0.04$ & $105.37 \pm 0.04$ & 17.55 \\
\hline Nigella glandulifera Freyn et Sint. & $\mathrm{SD}$ & $78.56 \pm 0.01$ & $95.88 \pm 0.04$ & $2.58 \pm 0.01$ & $113.01 \pm 0.01$ & 10.05 \\
\hline Oldenlandia diffusa (Willd.) Roxb. & HR & $43.62 \pm 0.02$ & $62.44 \pm 0.05$ & $4.12 \pm 0.01$ & $69.83 \pm 0.02$ & 11.58 \\
\hline Ophiopogon japonicus (L.f.) Ker-Gawl. & $\mathrm{RT}$ & $9.31 \pm 0.01$ & $65.68 \pm 0.05$ & $0.62 \pm 0.01$ & $96.24 \pm 0.01$ & 39.34 \\
\hline Paeonia veitchii Lynch & RT & $61.27 \pm 0.05$ & $49.03 \pm 0.01$ & $2.95 \pm 0.01$ & $101.88 \pm 0.03$ & 22.17 \\
\hline Paeonia lactiflora Pall. & RT & $-8.32 \pm 0.01$ & $77.45 \pm 0.01$ & $1.16 \pm 0.01$ & $98.85 \pm 0.03$ & 16.01 \\
\hline Paeonia suffruticosa Andr. & BK & $31.64 \pm 0.04$ & $64.88 \pm 0.02$ & $4.15 \pm 0.01$ & $70.23 \pm 0.01$ & 28.7 \\
\hline Panax ginseng C. A. Mey. & SR & $26.73 \pm 0.04$ & $71.25 \pm 0.06$ & $0.92 \pm 0.01$ & $101.26 \pm 0.01$ & 36.617 \\
\hline Perilla frutescens (L.) Britt. & $\mathrm{HR}$ & $11.22 \pm 0.05$ & $64.07 \pm 0.02$ & $2.23 \pm 0.01$ & $103.16 \pm 0.01$ & 12.36 \\
\hline Peucedanum praeruptorum Dunn & RT & $66.44 \pm 0.02$ & $102.58 \pm 0.17$ & $4.51 \pm 0.01$ & $20.67 \pm 0.07$ & 13.07 \\
\hline Polygonatum odoratum (Mill.) Druce & ST & $-3.64 \pm 0.02$ & $47.88 \pm 0.01$ & $1.00 \pm 0.01$ & $97.64 \pm 0.01$ & 32.28 \\
\hline Polygonum multiflorum Thunb. & RT & $36.49 \pm 0.02$ & $63.84 \pm 0.02$ & $4.91 \pm 0.01$ & $73.7 \pm 0.02$ & 12.57 \\
\hline Poria $\operatorname{cocos}$ (Schw.) Wolf & SC & $56.75 \pm 0.04$ & $12.61 \pm 0.06$ & $1.34 \pm 0.01$ & $49.13 \pm 0.03$ & 2.21 \\
\hline Psoralea corylifolia $\mathrm{L}$. & FR & $41.35 \pm 0.04$ & $93.39 \pm 0.04$ & $1.16 \pm 0.01$ & $7.93 \pm 0.01$ & 5.34 \\
\hline Pueraria lobata (Willd.) Ohwi & RT & $58.64 \pm 0.03$ & $93.10 \pm 0.08$ & $0.68 \pm 0.01$ & $101.30 \pm 0.02$ & 20.25 \\
\hline Pyrola calliantha $\mathrm{H}$. Andres. & $\mathrm{HR}$ & $20.09 \pm 0.07$ & $50.68 \pm 0.02$ & $3.04 \pm 0.01$ & $106.48 \pm 0.04$ & 11.6 \\
\hline Rehmannia glutinosa Libosch. & RT & $-14.78 \pm 0.01$ & $38.15 \pm 0.01$ & $0.45 \pm 0.01$ & $96.41 \pm 0.01$ & 39.67 \\
\hline Rosa laevigata Michx. & FR & $29.37 \pm 0.02$ & $69.39 \pm 0.03$ & $4.40 \pm 0.01$ & $91.48 \pm 0.06$ & 22.8 \\
\hline Rubia cordifolia L. & SR & $69.99 \pm 0.03$ & $113.22 \pm 0.12$ & $5.30 \pm 0.01$ & $102.03 \pm 0.06$ & 12.67 \\
\hline Salvia miltiorrhiza Bge. & SR & $7.35 \pm 0.01$ & $82.25 \pm 0.14$ & $2.02 \pm 0.01$ & $100.35 \pm 0.01$ & 40.42 \\
\hline Santalum album $\mathrm{L}$. & HW & $36.59 \pm 0.02$ & $61.80 \pm 0.03$ & $4.61 \pm 0.01$ & $63.65 \pm 0.16$ & 7.25 \\
\hline Saposhnikovia divaricata (Turcz.) Schischk. & RT & $6.73 \pm 0.01$ & $56.66 \pm 0.01$ & $3.02 \pm 0.01$ & $92.08 \pm 0.10$ & 20.51 \\
\hline Scutellaria baicalensis Georgi & $\mathrm{RT}$ & $23.55 \pm 0.01$ & $69.68 \pm 0.01$ & $3.07 \pm 0.01$ & $100.93 \pm 0.01$ & 47.06 \\
\hline Scutellaria barbata D. Don & HR & $53.51 \pm 0.03$ & $98.59 \pm 0.03$ & $4.28 \pm 0.01$ & $101.75 \pm 0.04$ & 21.39 \\
\hline Satsstrea japonica (Thunb.) De. & $\mathrm{BK}$ & $70.55 \pm 0.01$ & $126.05 \pm 0.14$ & $4.19 \pm 0.01$ & $91.61 \pm 0.03$ & 4.66 \\
\hline Spatholobus suberectus Dunn. & ST & $33.79 \pm 0.01$ & $27.24 \pm 0.01$ & $4.98 \pm 0.01$ & $92.21 \pm 0.07$ & 16.07 \\
\hline Stephania tetrandra S. Moore & RT & $52.29 \pm 0.06$ & $8.38 \pm 0.01$ & $2.06 \pm 0.01$ & $98.80 \pm 0.03$ & 11.03 \\
\hline Tribulus terrestris $\mathrm{L}$. & FR & $73.48 \pm 0.02$ & $71.80 \pm 0.09$ & $4.15 \pm 0.01$ & $87.66 \pm 0.05$ & 8.44 \\
\hline Trichosanthes kirilowii Maxim. & $\mathrm{PE}$ & $-2.38 \pm 0.03$ & $29.54 \pm 0.01$ & $1.07 \pm 0.01$ & $101.00 \pm 0.01$ & 35.97 \\
\hline Typha angustifolia L. & PL & $78.99 \pm 0.05$ & $48.80 \pm 0.01$ & $3.66 \pm 0.01$ & $85.85 \pm 0.02$ & 7.09 \\
\hline Typhonium giganteum Engl. & ST & $7.41 \pm 0.01$ & $47.14 \pm 0.01$ & $0.46 \pm 0.01$ & $94.68 \pm 0.02$ & 24.56 \\
\hline Xanthium sibiricum Patr. & $\mathrm{HR}$ & $76.34 \pm 0.04$ & $94.41 \pm 0.07$ & $4.46 \pm 0.01$ & $83.82 \pm 0.06$ & 5.73 \\
\hline Zingiber officinale Rosc. & SR & $91.28 \pm 0.01$ & $98.31 \pm 0.05$ & $2.20 \pm 0.01$ & $41.37 \pm 0.04$ & 10.10 \\
\hline $\mathrm{L}-\mathrm{NIL}^{\mathrm{g}}$ & & $35.2 \pm 0.01$ & $84.29 \pm 0.01$ & $3.22 \pm 0.01$ & $99.95 \pm 0.03$ & \\
\hline
\end{tabular}

${ }^{\mathrm{a}} \mathrm{HR}$ : herb; RT: root; ST: stem; LF: leaf; TW: twig; FL: flower; FR: fruit; SD: seed; SC: sclerotium; HW: heartwood; SR: stem and root; PE: pericarp.

${ }^{\mathrm{b}}$ Percent inhibition of NO production: Griess reaction was carried out to measure the production of nitrite in LPS/IFN $\gamma$-stimulated RAW264.7 cells in the absence or presence of $100 \mu \mathrm{g} / \mathrm{mL}$ herb extracts.

${ }^{\mathrm{c}}$ Cell growth: MTT was performed to measure cell growth. The growth rate of control (no herb extract treatment) was considered as $100 \%$.

${ }^{\mathrm{d}} \mathrm{NO}$ production: Griess reaction was used to measure the amount of nitrite in unstimulated RAW264.7 cells in the absence and presence of $100 \mu \mathrm{g} / \mathrm{mL}$ herb extracts.

${ }^{\mathrm{e}}$ Cell cytotoxicity: MTT assay was performed to determine cell cytotoxicity of unstimulated RAW264.7 cells treated with herb extracts. Untreated group was considered as $100 \%$.

${ }^{\mathrm{f}}$ Percent yield of extract obtained from $70 \%$ ethanol extraction of each $100 \mathrm{~g}$ dry herb.

${ }^{\mathrm{g}}$ Percent inhibition of iNOS activity at the test concentration of $50 \mu \mathrm{M}$. 
TABLE 2: Characteristics (flavor, nature, and meridian distributions) of the 10 most potent anti-inflammatory herbs.

\begin{tabular}{lccc}
\hline Plant name and authority & Flavors $^{\mathrm{a}, \mathrm{b}}$ & Natures $^{\mathrm{a}, \mathrm{b}}$ & Meridian distributions $^{\mathrm{a}, \mathrm{b}}$ \\
\hline Daphne genkwa Sieb.et Zucc. & Bitter, pungent & Warm & Lung, spleen, kidney \\
Caesalpinia sappan L. & Sweat, salty & Moderate & Heart, liver, spleen \\
Forsythia suspensa (Thunb.) Vahl & Bitter & Litter cold & Lung, heart, intestinum tenue \\
Zingiber officinale Rosc. & Pungent & Hot & Spleen, stomach, kidney, heart, lung \\
Inula japonica Thunb. & Bitter, pungent, salty & Little warm & Lung, spleen, stomach, intestinum crassum \\
Ligusticum chuanxiong Hort. & Pungent & Warm & Liver, gallbladder, pericardium meridian \\
Curcuma longa L. & Pungent, bitter & Warm & Spleen, liver \\
Typha angustifolia L. & Sweat & Moderate & Liver, pericardium meridian \\
Nigella glandulifera Freyn et Sint. & Sweat, pungent & Warm & Liver, kidney \\
Bletilla striata (Thunb.) Reichb.f. & Bitter, sweet, astringent & Little cold & Lung, liver, stomach \\
\hline
\end{tabular}

${ }^{a}$ Based on Chinese Pharmacopoeia (2010).

${ }^{\mathrm{b}}$ Based on Chinese Materia Medica (1998).

TABLE 3: Percentage distribution of herbs with the ability to inhibit over $50 \%$ NO production in each TCM characteristics.

\begin{tabular}{|c|c|c|c|c|}
\hline TCM characteristic & $\begin{array}{c}\text { Hit extracts } \\
\text { (inhibition over } 50 \% \text { ) }\end{array}$ & $\begin{array}{c}\text { Percentage of } \\
\text { effective herbs (32) }\end{array}$ & $\begin{array}{c}\text { Herbs } \\
\text { sharing same } \\
\text { flavors }\end{array}$ & $\begin{array}{l}\text { Percentage } \\
\text { (in } 81 \text { herbs) }\end{array}$ \\
\hline \multicolumn{5}{|l|}{ Four properties } \\
\hline Cold & 9 & 28.13 & 30 & 37.04 \\
\hline Cool & 1 & 3.13 & 2 & 2.47 \\
\hline Warm & 11 & 34.38 & 33 & 40.74 \\
\hline Hot & 3 & 9.38 & 4 & 4.94 \\
\hline Moderate & 8 & 25 & 12 & 14.81 \\
\hline \multicolumn{5}{|l|}{ Five flavors } \\
\hline Pungent & 20 & 62.5 & 42 & 51.85 \\
\hline Sweet & 9 & 28.13 & 30 & 37.04 \\
\hline Bitter & 20 & 62.5 & 47 & 58.02 \\
\hline Sour & 0 & 0 & 3 & 3.70 \\
\hline Astringent & 2 & 6.25 & 6 & 7.41 \\
\hline Salty & 3 & 9.38 & 3 & 3.70 \\
\hline Mild & 2 & 6.25 & 3 & 3.70 \\
\hline \multicolumn{5}{|l|}{ Meridian distributions } \\
\hline Liver & 18 & 56.25 & 43 & 53.09 \\
\hline Lung & 17 & 53.13 & 35 & 43.21 \\
\hline Spleen & 13 & 40.63 & 29 & 35.80 \\
\hline Heart & 10 & 31.25 & 30 & 37.04 \\
\hline Kidney & 8 & 25 & 25 & 30.86 \\
\hline Stomach & 7 & 21.88 & 22 & 27.16 \\
\hline Intestinum crassum & 4 & 12.5 & 9 & 11.11 \\
\hline Urinary bladder & 2 & 6.25 & 7 & 8.64 \\
\hline Gallbladder & 2 & 6.25 & 6 & 7.41 \\
\hline Intestinum tenue & 1 & 3.13 & 2 & 2.47 \\
\hline
\end{tabular}

common characteristics that are of pungent/bitter flavor, warm nature, and lung/liver meridian.

\subsection{Correlation between Cell Protective Effect and TCM} Characteristics of Herbs. Chronic inflammation often leads to cell damage and thus agents capable of deterring this process are actively sought. Examining 21 herbs with the
TCM characteristic of pungent flavor, we observed that, under the costimulation of LPS and IFN $\gamma$, RAW264.7 cells treated with these herb extracts displayed $90 \%$ increase in cell viability (Table 4). Moreover, herbs with pungent flavor also conferred the highest degree of cell protection in LPS/IFN $\gamma$ stimulated cells in comparison with herbs with other flavors (Figure 1). 
TABLE 4: Percentage distribution of herbs with cell protective capability in each TCM characteristics.

\begin{tabular}{|c|c|c|c|c|}
\hline TCM characteristics & Hit herbs $^{\mathrm{a}}$ & Percentage (21 herbs) & Hit herbs $^{\mathrm{b}}$ & Percentage (43 herbs) \\
\hline \multicolumn{5}{|l|}{ Four natures } \\
\hline Cold & 5 & 23.81 & 19 & 44.19 \\
\hline Cool & 1 & 4.76 & 1 & 2.33 \\
\hline Moderate & 5 & 23.81 & 5 & 11.63 \\
\hline Warm & 8 & 38.10 & 15 & 34.88 \\
\hline Hot & 2 & 9.52 & 1 & 2.33 \\
\hline \multicolumn{5}{|l|}{ Five flavors } \\
\hline Pungent & 15 & 71.43 & 21 & 48.84 \\
\hline Sweet & 9 & 42.86 & 14 & 32.56 \\
\hline Bitter & 13 & 61.90 & 22 & 51.16 \\
\hline Sour & 0 & 0 & 0 & 0 \\
\hline Astringent & 2 & 9.52 & 2 & 4.65 \\
\hline Salty & 2 & 9.52 & 1 & 2.33 \\
\hline Mild & 0 & 0 & 2 & 4.651 \\
\hline \multicolumn{5}{|l|}{ Meridian distributions } \\
\hline Liver & 9 & 42.86 & 20 & 46.51 \\
\hline Lung & 11 & 52.38 & 18 & 41.86 \\
\hline Spleen & 10 & 47.62 & 12 & 27.91 \\
\hline Heart & 8 & 38.10 & 14 & 32.56 \\
\hline Kidney & 8 & 38.10 & 12 & 27.91 \\
\hline Stomach & 4 & 19.05 & 13 & 30.23 \\
\hline Intestinum crassum & 3 & 14.29 & 3 & 6.977 \\
\hline Urinary bladder & 1 & 4.76 & 6 & 13.95 \\
\hline Gallbladder & 0 & 0 & 5 & 11.63 \\
\hline Intestinum tenue & 0 & 0 & 1 & 2.326 \\
\hline
\end{tabular}

${ }^{a}$ Herbs with over $90 \%$ cell protective capability in stimulated RAW264.7 cells.

${ }^{\mathrm{b}}$ Herbs with ability to increase over $90 \%$ cell proliferation in resting RAW264.7 cells.

\section{Discussion}

Overproduction of NO due to the elevated iNOS expression has been convincingly linked to the pathogenesis of chronic inflammation and cancer [34]. Hence, agents that can selectively suppress iNOS-generated NO production should be effective to treat chronic inflammation and to prevent cancer. In fact, recent studies demonstrate that selective iNOS inhibitors L-NIL and $1400 \mathrm{~W}$ are therapeutically effective as anti-inflammation and anticancer drugs [35, 36].

Macrophages play a critical role in regulating inflammation. Macrophages are activated by external stimuli and activated macrophages produce various inflammatory mediators such as NO and reactive oxygen species. Chinese herbs are the rich sources for anti-inflammatory agents and efforts have been made to identify effective components in these herbs $[37,38]$. Taking advantage of the well-established RAW264.7 cell model, we evaluated 81 herb extracts for their inhibitory effect on LPS/IFN $\gamma$-induced NO production. Among them, the extract of Daphne genkwa Sieb.et Zucc showed the strongest inhibitory effect on NO production. The constituents isolated from Daphne genkwa Sieb.et Zucc were previously reported to provoke cytotoxic effect to various tumor cell lines and to suppress outgrowth of transplanted

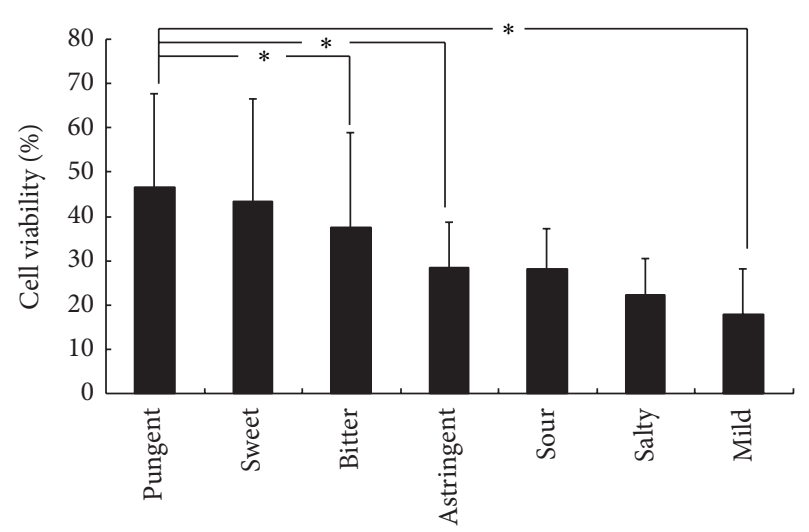

FIgURE 1: Comparison of herbs with cell viability in different flavors. Average of cell viability of LPS/IFN $\gamma$-stimulated RAW264.7 cells treated with herbs belonging to different flavor. ${ }^{*} P<0.05$.

mouse sarcoma S180 in mice [39]. We speculate that anticancer effect of Daphne genkwa Sieb.et Zucc may be functionally associated with its anti-inflammatory capability. In our study, we found that Rubia cordifolia L. and several others decrease LPS/IFN $\gamma$-induced NO production without causing significant cytotoxicity to RAW264.7 cells. These herbs may 
thus be promising candidates as effective drugs to control inflammation and cancer. Although it is currently unclear how these extracts block LPS/IFN-induced NO production in RAW264.7 cells, the finding that Mollugin suppresses the inflammatory response by blocking the Janus kinase-signal transducers and activators of transcription signaling pathway [40] implicates that herbs may target the different steps of the signaling cascade mediating LPS/IFN-induced NO production to exert their anti-inflammatory roles.

Based on the theory of TCM, we classified these 81 herbs according to distinct flavors (pungent, sweet, sour, bitter, astringent, salty, or mild), natures (cold, cool, moderate, warm, or hot), and meridian distributions (liver, kidney, heart, spleen, etc.). Our study showed that the TCM characteristic of flavor correlated very well with the potency to inhibit NO production-pungent flavor is the strongest, bitter is slightly weaker than pungent, sweet flavors is intermediate, and astringent, salty, mild, or sour flavor is weak or not effective. TCM characteristics of nature and meridian distribution are also associated with the potency to inhibit NO production. For instance, higher pe of herbs with the capability to block NO production has the characteristics of warm nature. Characteristics of liver and lung meridians are the major meridian distributions found in herbs whose extracts can block $50 \%$ of NO production. Taken together, we reason that TCM characteristics can potentially be very useful to guide the search for effective anti-inflammatory agents in Chinese herbs.

TCM characteristic is a systematic expression of the distinct property elicited by Materia Medica in humans. Theory of flavors in TCM constitutes the core context of Chinese herb usage guidance. In TCM, the characteristic of flavor is the combination of both real taste and curative effect. According to Shen Nong Ben Cao Jing (Shennong's Classic of Material Medica), an important TCM book firstly written on Chinese herbal flavor and property theory, pungent flavor, which is related to lung meridian, can disperse the internal heat with sudorifics which in turn promote the circulation of Qi and blood. Herbs with pungent flavor have actually been used for thousand years in China to invigorate the circulation of blood and break the block of Qi. The fact that inflammation-related diseases are associated with the symptom of Qi and blood blockage may explain the effectiveness of herbs with pungent flavor to suppress inflammation.

Our study was limited to the investigation of 81 herb extracts on their effect on LPS/IFN $\gamma$-induced NO production and cell growth in macrophage RAW264.7 cells. The results generated from this study nevertheless support a close association between modern pharmacology/biomedical science and TCM theory. TCM theory was developed based on thousand years of clinical experience, and the material and pharmacological basis of TCM remains to be explained by the modern biomedical science. We believe that this study has contributed toward this goal.

\section{Conflict of Interests}

The authors declare that there is no conflict of interests regarding the publication of this paper.

\section{Acknowledgments}

This work was supported by the National Science and Technology Major Project of the Ministry of Science and Technology of China (2009ZX09311-003), the Young Scientists Fund of the National Natural Science Foundation of China (81001666), Innovation Program of Shanghai Municipal Education Commission (13YZ048), and the Foundation of Shanghai Education Commission for Outstanding Young Teachers in University (SZY07029).

\section{References}

[1] G. Y. Chen and G. Nuñez, "Sterile inflammation: sensing and reacting to damage," Nature Reviews Immunology, vol. 10, no. 12, pp. 826-837, 2010.

[2] R. Ross, "Atherosclerosis-an inflammatory disease," The New England Journal of Medicine, vol. 340, no. 2, pp. 115-126, 1999.

[3] H. L. Weiner and D. Frenkel, "Immunology and immunotherapy of Alzheimer's disease," Nature Reviews Immunology, vol. 6, no. 5, pp. 404-416, 2006.

[4] C. Garcia, B. Feve, P. Ferré et al., "Diabetes and inflammation: fundamental aspects and clinical implications," Diabetes and Metabolism, vol. 36, no. 5, pp. 327-338, 2010.

[5] L. M. Coussens and Z. Werb, "Inflammation and cancer," Nature, vol. 420, no. 6917, pp. 860-867, 2002.

[6] H. Clevers, "At the crossroads of inflammation and cancer," Cell, vol. 118, no. 6, pp. 671-674, 2004.

[7] A. Mantovani, P. Allavena, A. Sica, and F. Balkwill, "Cancerrelated inflammation," Nature, vol. 454, no. 7203, pp. 436-444, 2008.

[8] C. J. Lowenstein and E. Padalko, "iNOS (NOS2) at a glance," Journal of Cell Science, vol. 117, no. 14, pp. 2865-2867, 2004.

[9] C. V. Suschek, O. Schnorr, and V. Kolb-Bachofen, "The role of iNOS in chronic inflammatory processes in vivo: is it damagepromoting, protective, or active at all?" Current Molecular Medicine, vol. 4, no. 7, pp. 763-775, 2004.

[10] G. C. Brown, "Mechanisms of inflammatory neurodegeneration: iNOS and NADPH oxidase," Biochemical Society Transactions, vol. 35, no. 5, pp. 1119-1121, 2007.

[11] H. H. Schmidt and U. Walter, "NO at work," Cell, vol. 78, no. 6, pp. 919-925, 1994.

[12] K. Bian and F. Murad, "Nitric oxide (NO)-biogeneration, regulation, and relevence to human diseases," Frontiers in Bioscience, vol. 8, pp. d264-d278, 2003.

[13] H. Ohshima and H. Bartsch, "Chronic infections and inflammatory processes as cancer risk factors: possible role of nitric oxide in carcinogenesis," Mutation Research, vol. 305, no. 2, pp. 253-264, 1994.

[14] J. R. Kanwar, R. K. Kanwar, H. Burrow, and S. Baratchi, "Recent advances on the roles of NO in cancer and chronic inflammatory disorders," Current Medicinal Chemistry, vol. 16, no. 19, pp. 2373-2394, 2009.

[15] S. Heemskerk, R. Masereeuw, F. G. Russel, and P. Pickkers, "Selective iNOS inhibition for the treatment of sepsis-induced acute kidney injury," Nature Reviews Nephrology, vol. 5, no. 11, pp. 629-640, 2009.

[16] V. Kostourou, J. E. Cartwright, A. P. Johnstone et al., "The role of tumour-derived iNOS in tumour progression and angiogenesis," British Journal of Cancer, vol. 104, no. 1, pp. 8390, 2011. 
[17] C. Hesslinger, A. Strub, R. Boer, W.-R. Ulrich, M. D. Lehner, and C. Braun, "Inhibition of inducible nitric oxide synthase in respiratory diseases," Biochemical Society Transactions, vol. 37, no. 4, pp. 886-891, 2009.

[18] M. Lechner, P. Lirk, and J. Rieder, "Inducible nitric oxide synthase (iNOS) in tumor biology: the two sides of the same coin," Seminars in Cancer Biology, vol. 15, no. 4, pp. 277-289, 2005.

[19] B. Fitzpatrick, M. Mehibel, R. L. Cowen, and I. J. Stratford, "iNOS as a therapeutic target for treatment of human tumors," Nitric Oxide, vol. 19, no. 2, pp. 217-224, 2008.

[20] G.-Y. Yang, S. Taboada, and J. Liao, "Induced nitric oxide synthase as a major player in the oncogenic transformation of inflamed tissue," Methods in Molecular Biology, vol. 512, pp. 119156, 2009.

[21] C. M. Ulrich, J. Bigler, and J. D. Potter, "Non-steroidal antiinflammatory drugs for cancer prevention: promise, perils and pharmacogenetics," Nature Reviews Cancer, vol. 6, no. 2, pp. 130-140, 2006.

[22] E. J. Jacobs, "Will an aspirin a day help keep fatal cancer away?" The Lancet, vol. 377, no. 9759, pp. 3-4, 2011.

[23] R. Basha, C. H. Baker, U. T. Sankpal et al., "Therapeutic applications of NSAIDS in cancer: special emphasis on tolfenamic acid," Frontiers in Bioscience, vol. 3, pp. 797-805, 2011.

[24] B. B. Aggarwal, R. V. Vijayalekshmi, and B. Sung, "Targeting inflammatory pathways for prevention and therapy of cancer: short-term friend, long-term foe," Clinical Cancer Research, vol. 15, no. 2, pp. 425-430, 2009.

[25] A. Murakami and H. Ohigashi, “Targeting NOX, INOS and COX-2 in inflammatory cells: chemoprevention using food phytochemicals," International Journal of Cancer, vol. 121, no. 11, pp. 2357-2363, 2007.

[26] S. Bengmark, "Curcumin, an atoxic antioxidant and natural NF$\kappa \mathrm{B}$, cyclooxygenase-2, lipooxygenase, and inducible nitric oxide synthase inhibitor: a shield against acute and chronic diseases," Journal of Parenteral and Enteral Nutrition, vol. 30, no. 1, pp. 4551, 2006.

[27] V. B. Patel, S. Misra, B. B. Patel, and A. P. Majumdar, "Colorectal cancer: chemopreventive role of curcumin and resveratrol," Nutrition and Cancer, vol. 62, no. 7, pp. 958-967, 2010.

[28] C. H. Hong, S. K. Hur, O.-J. Oh, S. S. Kim, K. A. Nam, and S. K. Lee, "Evaluation of natural products on inhibition of inducible cyclooxygenase (COX-2) and nitric oxide synthase (iNOS) in cultured mouse macrophage cells," Journal of Ethnopharmacology, vol. 83, no. 1-2, pp. 153-159, 2002.

[29] A. Murakami, "Chemoprevention with phytochemicals targeting inducible nitric oxide synthase," Forum of Nutrition, vol. 61, pp. 193-203, 2009.

[30] C. Cerella, C. Sobolewski, M. Dicato, and M. Diederich, "Targeting COX-2 expression by natural compounds: a promising alternative strategy to synthetic COX-2 inhibitors for cancer chemoprevention and therapy," Biochemical Pharmacology, vol. 80, no. 12, pp. 1801-1815, 2010.

[31] H. Liao, L. K. Banbury, and D. N. Leach, "Antioxidant activity of 45 Chinese herbs and the relationship with their TCM characteristics," Evidence-Based Complementary and Alternative Medicine, vol. 5, no. 4, pp. 429-434, 2008.

[32] L. C. Green, D. A. Wagner, J. Glogowski, P. L. Skipper, J. S. Wishnok, and S. R. Tannenbaum, "Analysis of nitrate, nitrite, and $[15 \mathrm{~N}]$ nitrate in biological fluids," Analytical Biochemistry, vol. 126, no. 1, pp. 131-138, 1982.
[33] B. Ayissi Owona, N. F. Njayou, S. Laufer, P. F. Moundipa, and H. J. Schluesener, "A fraction of stem bark extract of Entada africana suppresses lipopolysaccharide-induced inflammation in RAW 264.7 cells," Journal of Ethnopharmacology, vol. 149, no. 1, pp. 162-168, 2013.

[34] Y. Wu, S. Antony, J. L. Meitzler, and J. H. Doroshow, "Molecular mechanisms underlying chronic inflammation-associated cancers," Cancer Letters, 2013.

[35] A. G. Sikora, A. Gelbard, M. A. Davies et al., "Targeted inhibition of inducible nitric oxide synthase inhibits growth of human melanoma in vivo and synergizes with chemotherapy," Clinical Cancer Research, vol. 16, no. 6, pp. 1834-1844, 2010.

[36] K. Engels, S. K. Knauer, S. Loibl et al., "NO signaling confers cytoprotectivity through the survivin network in ovarian carcinomas," Cancer Research, vol. 68, no. 13, pp. 5159-5166, 2008.

[37] K. S. Kim, X. Cui, D. S. Lee et al., "Anti-inflammatory effect of neoechinulin A from the marine fungus Eurotium sp. SF-5989 through the suppression of NF- $\kappa$ B and p38 MAPK pathways in lipopolysaccharide-stimulated RAW264.7 macrophages," Molecules, vol. 18, no. 11, pp. 13245-13259, 2013.

[38] H. J. Lee, Y. J. Jeong, T. S. Lee et al., "Moringa fruit inhibits LPSinduced NO/iNOS expression through suppressing the NF- $\kappa \mathrm{B}$ activation in RAW264.7 cells," The American Journal of Chinese Medicine, vol. 41, no. 5, pp. 1109-11023, 2013.

[39] S. Li, G. Chou, Y. Hseu, H. Yang, H. Kwan, and Z. Yu, "Isolation of anticancer constituents from flos genkwa (Daphne genkwa Sieb.et Zucc.) through bioassay-guided procedures," Chemistry Central Journal, vol. 7, article 159, 2013.

[40] Z. G. Zhu, H. Jin, P. J. Yu, Y. X. Tian, J. J. Zhang, and S. G. Wu, "Mollugin inhibits the inflammatory response in lipopolysaccharide-stimulated RAW264.7 macrophages by blocking the Janus kinase-signal transducers and activators of transcription signaling pathway," Biological \& Pharmaceutical Bulletin, vol. 36, no. 3, pp. 399-406, 2013. 


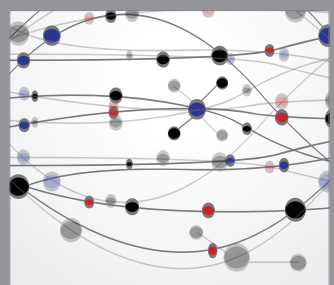

The Scientific World Journal
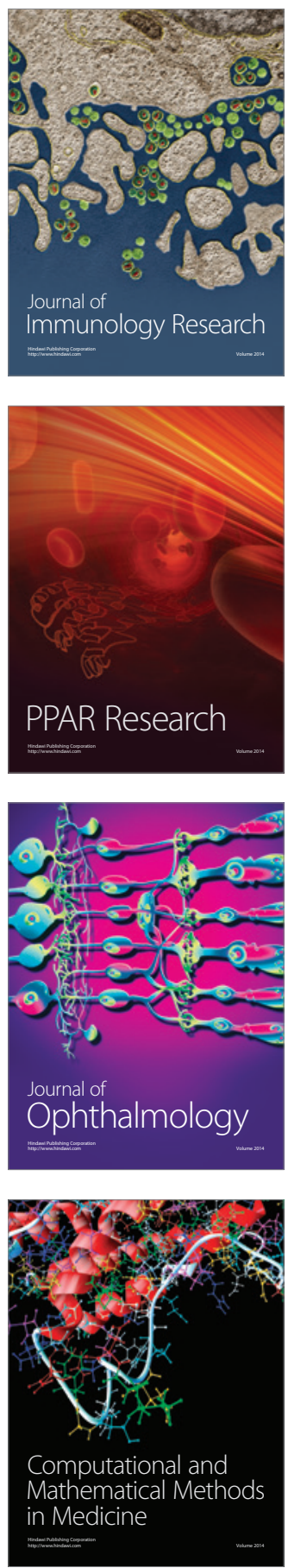

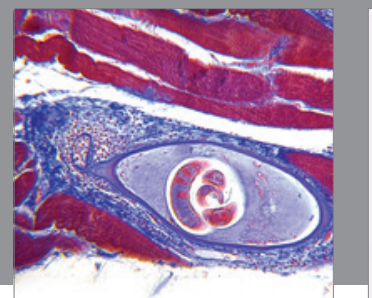

Gastroenterology

Research and Practice
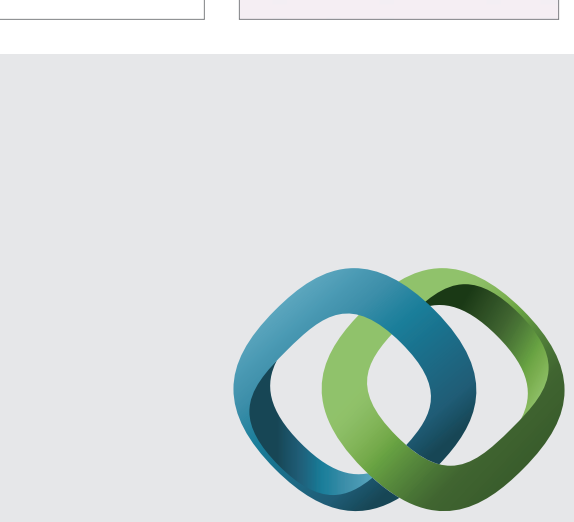

\section{Hindawi}

Submit your manuscripts at

http://www.hindawi.com
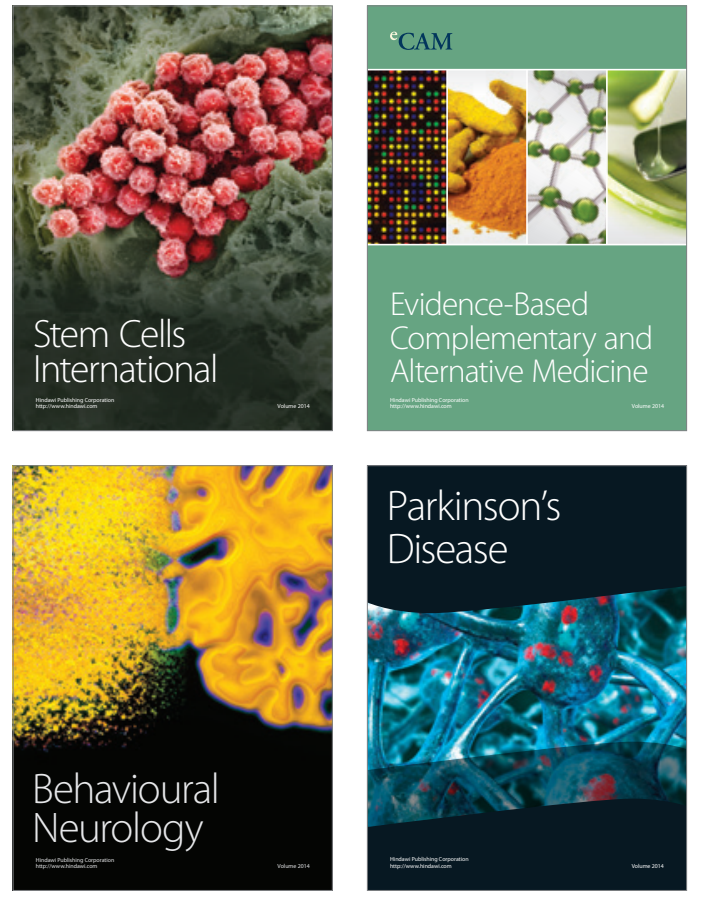
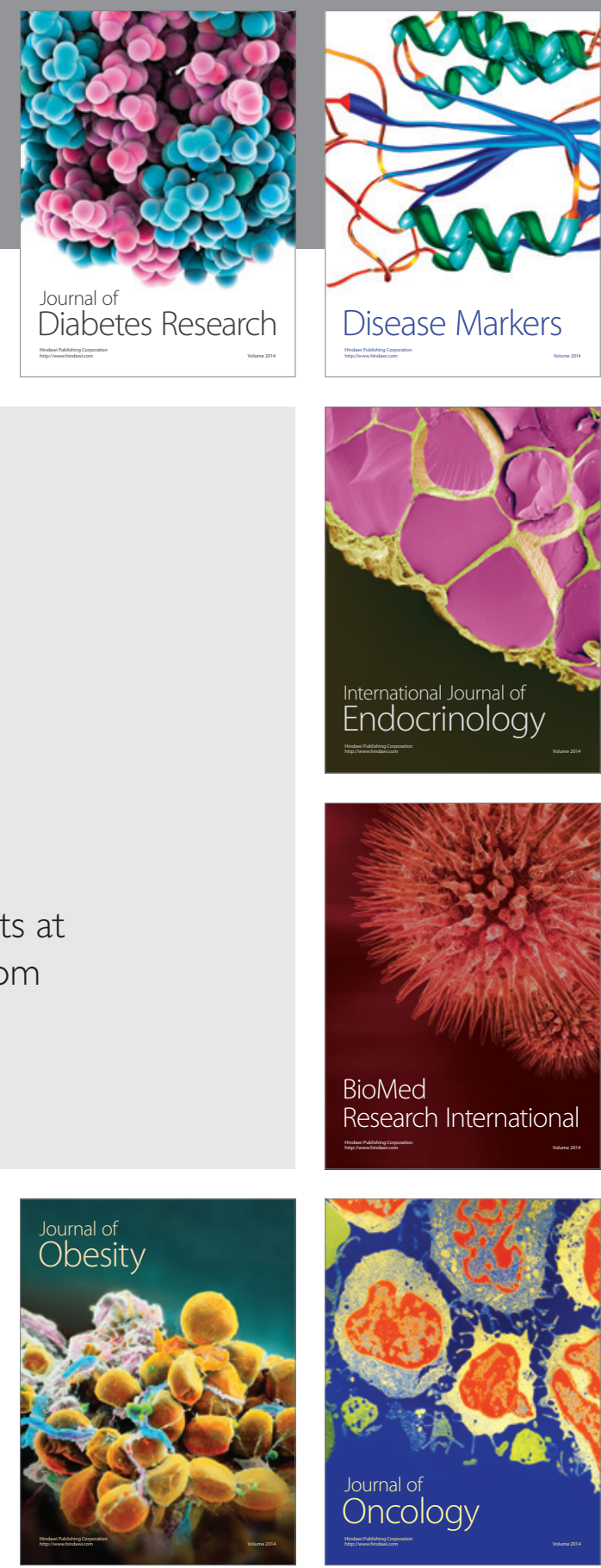

Disease Markers
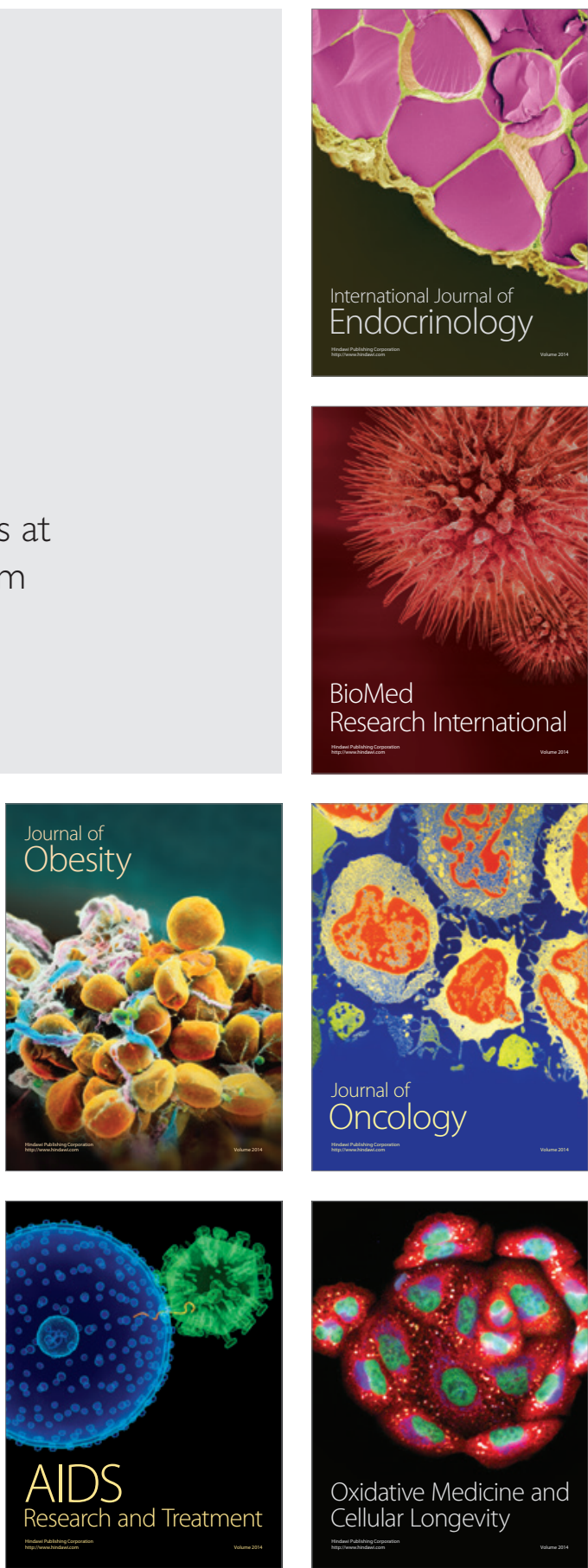